\title{
La Educación Física y el conocimiento que ésta promueve
}

\author{
Physical education and the knowledge it promotes
}

Luis Felipe Brito Soto

\section{Resumen}

Hace algún tiempo, directivos, docentes, alumnos y padres de familia han considerado la educación física como valiosa. Sin embargo, el estatus educativo, desde una perspectiva social, la pone comparativamente con otras asignaturas (actividades tecnológicas, artisticas) en el último peldaño de la escala educativa.

En vez de ubicar la educación fisica entre los primeros lugares, el debate que presento pretende más bien señalar que la actividad corporal tiene mucho que ofrecer en el proceso de aprender y en la construcción del conocimiento. Mi aportación tiene como finalidad proporcionar a los docentes argumentos pedagógicos que los ayuden en su tarea educativa.

Hoy los profesionales de esta asignatura deben eliminar la idea de que esta especialidad sólo tiene que ver con "las capacidades fisicas", que son los prácticos, que la intervención se reduce a lo corporal, que sólo interesan los aspectos del rendimiento, lo cuantificable.

El avance en la investigación en torno del aprendizaje ha demostrado la pertinencia que la acción corporal tiene para ello, y considera la motricidad como un sistema de actuación de tipo inteligente. Por tanto, es importante, quizás urgente, reconocer que el trabajo en el patio de la escuela tiene que ver con cuerpos que se mueven, que se mueven, si, pero con idea, con creatividad, con intención, con voluntad, que dan sentido a la semiótica corporal. Esto lo sentenció Wallon en 1942 en su libro Del acto al pensamiento.

En vez de repasar todos y cada uno de los tratados que hoy asignan al trabajo corporal una orientación pedagógica y didáctica distinta, si conviene centrar la atención en uno de los planteamientos que considero más significativo.

Palabras clave: Conocimiento y motricidad, habilidades perceptivo-motrices, habilidades motrices básicas.

\section{Abstract}

Some time since, managers, teaching staff, student body and parents of a family have considered the physical education as a valuable discipline. However, from a social perspective, the educational status puts it comparatively with others subjects (technological activities, artistic activities) in the last step of the educational scale.

Instead of trying to place the physical education in the first steps, I present this debate in order to denote that the physical activity offers so much in the learning process and knowledge construction. My contribution has the purpose of providing for the teaching staff several pedagogical elements which help them in their educational task.

Today the physical education teachers must eliminate the idea that this specialty involves only "the physical capabilities", that these teachers are the skilled people, that the intervention is reduced to the physical exercises, that it is only interesting the performance and quantifiable aspects.

The progress in the investigation about the learning has shown the pertinence that the physical activity has for it, and considers the mobility as an intelligent performance system. Therefore, it is important, perhaps urgent, to recognize that the work in the school playground involves moving bodies, does involve moving bodies with idea, creativity, intention, will, and this gives sense to the bodily semiotics. These concepts were pointed out by Wallon in 1942 in his book From action to thought.

Instead of passing again all treatises that assign a different pedagogical, didactic orientation to the bodily work, it does be suitable to focus the attention on the approach I consider more significant.

Key words: $\quad$ Knowledge and mobility, perceptive-motive capabilities, basic motive capabilities.

Fecha de recepción: 27 de junio de 2005 .

Fecha de aceptación: 8 de agosto de 2005.

Profesor de Educación Física. Diplomado en diseño curricular por la Universidad Pedagógica Nacional. Diplomado en enseñanza superior por el Centro Pedagógico de la Universidad Autónoma de Chapingo. Actualmente es Subdirector de Educación Fisica en la Subsecretaria de Educación Básica de la Secretaria de Educación Pública. 


\section{Conocimiento y motricidad}

Arnold (1991) señala la existencia de dos tipos de conocimiento, que progresivamente han sido diferenciados, el saber qué y el saber cómo.

El saber qué se refiere al conocimiento de tipo teórico, como las matemáticas, la historia, la geografía y la ética, entre otras asignaturas. Es un conocimiento proposicional, es el más admitido y reconocido. Este saber se interesa por el descubrimiento de verdades respaldadas por la razón y confirmadas por la experiencia.

El saber cómo se interesa por la competencia activa, por el saber hacer, saber actuar. Tiene que ver con la ejecución y el logro de propósitos de acción, efectuados de maneras racio-nales y confirmadas por un grado razonable de éxito. Cuánto encierra la expresión: ¡Mira maestro...yo puedo hacerlo así!

$\mathrm{La}$ actividad física es conocimiento. Un conocimiento de carácter procedimental, tiene que ver con el saber cómo y tiene la misma importancia que el saber qué. Conviene advertir que estos dos tipos de conocimiento son racionales, válidos, y se complementan uno al otro.

Así, las acciones que la educación física promueve con los niños se dirigen a validar este tipo de conocimiento (el saber cómo) y a edificar la competencia motriz.

La competencia motriz es "el conjunto de conocimientos, procedimientos, actitudes y sentimientos que intervienen en las múltiples interacciones que realiza (el niño) en su medio y con los demás, y que permiten que los escolares superen los diferentes problemas motores planteados, tanto en las sesiones de educación física como en su vida diaria" (Ruiz, 1995, p. 19).

Esta competencia motriz hace referencia a un tipo de inteligencia respecto a las acciones, una inteligencia que supone conocer qué hacer, cómo hacerlo, cuándo y con quién actuar en función de las tareas planteadas. A la suma de la interacción de estos procesos, que se cristalizan en la incorporación del yo en la acción, lo denomino motricidad.

Ser competente supone poseer un repertorio de respuestas (en el caso que nos ocupa, respuestas motrices) de procedimientos para hacer $\mathrm{y}$ actuar, $\mathrm{y}$ de actitudes que le permiten al niño o niña una práctica autónoma sobre sus acciones. Ser competente implica también desarrollar el sentimiento de capacidad para actuar, de sentirse seguro, de poder salir airoso de los problemas, de perder miedos, de hacer propuestas, de hacer planes, de modificar y manifestar una actitud de alegría al ser causa de transformaciones en su medio (Harter en Ruiz, 1995, p. 20).
Lo valioso de este tipo de conocimiento que la motricidad promueve y de la competencia motriz que ésta edifica es que cuando una persona sabe cómo hacer las cosas, su conocimiento se halla actualizado y puesto en práctica en lo que hace; su inteligencia se manifiesta en sus propios hechos, destrezas y realizaciones, es decir, en su motricidad.

Ahora bien, ¿cómo promueve este tipo de conocimiento el profesional de educación física? Para contestar este interrogante hay que admitir, primero, que el docente proporciona a los niños (as) un contexto de práctica y, segundo, que ese contexto tiene mucho que ver con lo que aprenden o dejan de aprender los alumnos.

En contexto de práctica se encuentran tres elementos que configuran la intervención del maestro: a) la idea pedagógica; b) los contenidos educativos, y c) los estilos de enseñanza utilizados. A continuación presento sucintamente cada uno de ellos.

\section{La idea pedagógica}

La valoración del contenido y la forma de trabajo tienen que ver con las declaraciones pedagógicas tanto implícitas como explícitas que cada docente posee y van desde el reconocimiento de determinados contenidos, de los propósitos por lograr, hasta las relaciones interpersonales que se dan en los procesos de enseñanza y aprendizaje. En casos extremos, algunos docentes de la especialidad que valoran y creen en la clase modelo, el profesor ideal y los alumnos tipo. Mi argumentación no coincide con este caso; por el contrario, reconozco en la motricidad de los niños y las niñas un carácter inteligente, creativo, y emancipatorio.

La educación física de hoy ya no puede sostenerse en el concepto de hombre-máquina, es necesario dirigirse a la formación de un sujeto con capacidades, valores y actitudes, cuya movilidad tiene su punto original en los aspectos biológicos, psíquicos y sociológicos, los cuales han de tratarse desde la globalidad y la unidad del cuerpo, es decir, desde la acción motriz.

El movimiento corporal tiene distintas funciones: de conocimiento, anatómico-funcional, estética y expresiva, de comunicación y relación, higiénica, agonística, catártica y hedonista, y de compensación (Díaz, 1994, p. 60). La educación física debe atender no sólo una forma de movimiento, sino varias, de modo que satisfaga las distintas necesidades y expectativas de los niños y las niñas. La finalidad de la educación física en el ámbito escolar es impulsar y desarrollar a la persona dentro de 
una unidad en la que los procesos cog-nitivos y afectivos se relacionan con la acción y la mejora de las capacidades motrices de los alumnos, no sólo la acción y la obtención de un resultado competitivo de rendimiento. Es fundamental que la educación física ofrezca a los niños y las niñas la posibilidad de conocer su cuerpo y sus posibilidades de movimiento a través de un gran número de actividades corporales y motrices.

Entonces, si el movimiento corporal tiene distintas funciones, ¿por qué empeñarnos en desarrollar una sola? ¿por qué la apuesta es de modo predominante hacia los deportes?, ¿sólo el rendimiento es lo importante?

Sostengo, en definitiva, que la actividad educativa debe procurar el reconocimiento de la distinción corporal, donde el enriquecimiento de experiencias de relación sobrepase la eficiencia y la medición de los resultados. El saber cómo y el ser competente en el ámbito de la motricidad conllevan a un verdadero reconocimiento y aceptación de sí. En este sentido, coincido con Onofre:

...una educación física cuya forma natural de ser pase de la competición a la colaboración, para afirmar el propio cuerpo no como una oposición a los demás cuerpos, sino como aceptación de la propia identidad basada en la satisfacción de la actividad física como elemento de relación interpersonal y propiciadora de situaciones gratificantes, placenteras y saludables. (1998, p. 56)

Con base en los argumentos anteriores, la idea pedagógica y los fines de la actividad física escolar pueden ir por el siguiente camino, o bien vistos como propósitos pueden ser los siguientes:

\section{El desarrollo individual}

El ser humano dueño (señor) de sí mismo. Consiste en una práctica que enfatice el progreso que incorpore el sentimiento de autoestima, de autorrealización, de confianza en sí, que sus satisfacciones no estén sujetas sólo a la realización de un determinado patrón deportivo, sino de una práctica corporal global y recreativa. Ya no se debe sostener el triunfo de la actividad corporal por el hecho de encestar una pelota en el aro de baloncesto o al cronometraje de distancias en la carrera, la motricidad y la acción motriz tiene múltiples caminos y diversidad de patrones de movimiento.

\section{Adaptación al espacio, al medio físico}

El ser humano adaptado corporalmente a su entorno físico, al que controla. Se basa en el sentimiento de seguridad en la movilización del propio cuerpo en los distin- tos medios físicos, de un niño o niña que pierda miedos y sea seguro. Una adaptación del trabajo y la práctica de la acción motriz con base en las capacidades, los límites y las posibilidades de los niños y las niñas.

\section{Integración en el mundo social}

El ser humano relacionado con los demás. Se refiere a una integración de experiencias que se sostenga en principios pedagógicos, como "todos los niños pueden hacer las cosas", "todos tienen las mismas oportunidades", "todos han de participar". El fracaso escolar de la educación física y el sentimiento de incompetencia se deben, y en mucho, a la poca autoestima y valía personal promovidas por la acostumbrada selección de los dotados de aptitudes especiales, esta visión hay que desterrarla urgentemente. Los niños y las niñas necesitan una educación física de base. (González, 1993, p. 60).

\section{Acerca de los contenidos}

Los contenidos se refieren a qué se enseña, y se convierten en las actividades de aprendizaje. Lo que a continuación se apunta se presenta como parte del debate, y con el fin de orientar la práctica de la educación física. Los contenidos de la asignatura pueden orientarse por los siguientes campos:

\section{Desarrollo de las habilidades perceptivomotrices}

Una habilidad es "toda acción muscular o movimiento del cuerpo requerido para la ejecución con éxito de un acto deseado" (Singer en Contreras, 1998, p. 190), una habilidad supone un acto consciente e implica la edificación de la competencia motriz, posee dos características importantes: la flexibilidad ${ }^{1} \mathrm{y}$ adaptabilidad. La percepción, exige una experiencia más compleja que la mera sensación, es el resultado de los procesos de orden superior que integran y asocian a las sensaciones. Así, la percepción es "la adquisición del conocimiento del yo y del medio por selección y asociación de informaciones que, si bien comporta una importante parte innata, puede ser, sin embargo, objeto de aprendizaje" (Contreras, 1998, p. 181).

El contenido perceptivo-motor tiene que ver con el cúmulo de experiencias que la educación física da a los niños y niñas con el fin de que el docente les proporcione elementos para acrecentar su competencia motriz con base en el conocimiento de sí y en el reconocimiento de sus posibilidades de acción. Así, el niño o niña toma referencia del mundo físico que lo rodea, y le permite una mejor elaboración de esquemas o patrones 
de movimiento. Con este tipo de desarrollo se apuesta a que los niños y las niñas obtengan un mejoramiento en su habilidad perceptiva en todos sus aprendizajes.

\section{Desarrollo de habilidades motrices básicas}

Toda habilidad tiene un carácter evolucionista y parte de la carga filogenética de las personas, es decir, mediante el crecimiento, los patrones de movimiento van teniendo un grado cada vez mayor y mejor de ejecución y control. En la actualidad, muchos autores (Le Boulch, 1991; Contreras, 1998; Delgado, 1993; Devís, 1996) coinciden en que éstos son susceptibles de mejoramiento por medio de la maduración, la práctica y la experiencia. Las habilidades motrices básicas son los desplazamientos, los saltos, los giros y las manipulaciones.

El desarrollo de estas habilidades motrices básicas hace referencia a un trabajo de coordinación de tipo elemental (básico) que, por medio del ejercicio natural y cotidiano de caminar, trotar, correr, saltar, girar, lanzar, mediante una práctica vivenciada y basada en el juego, afirma los patrones de movimiento elementales y proporciona un mayor bagaje de experiencias que permite a los niños y niñas ampliar su motricidad, así como de experiencias del control y la incorporación del "yo" en la acción (competencia motriz).

\section{Juegos motores}

El juego es el principal interés de los niños en edad escolar. Por medio de éste aprenden el mundo físico, afectivo y social que les rodea. Es el fenómeno cultural más estudiado en la actualidad, por lo cual no insistiré en su valor. Pondré el acento en que el juego desarrolla el pensamiento estratégico y la lógica motriz y, precisamente, en estos elementos es donde gravita mi interés.

En el pensamiento estratégico y la lógica motriz se dan cita al menos tres fases: la percepción y el análisis de la situación y los sucesos del juego; la solución mental del problema o acción, y la solución y la respuesta motrices. Para consolidar este tipo de pensamiento y lógica de acción, es importante que el docente utilice un estilo de enseñanza no directivo, no debe emplear juegos que exijan modelos de movimiento y/o reglas determinados; por el contrario, debe dirigirse a formas de enseñanza que motiven la exploración y el descubrimiento donde los niños y las niñas puedan hacer propuestas y modificaciones a los juegos mismos.

Existen dos modelos de instrucción y enseñanza en educación física: el vertical y el horizontal. En el primero existe una fuerte tendencia por ejecutar patrones específicos y únicos de determinados deportes, es lo que comúnmente conocemos como la iniciación deportiva, y se acentúa más, por la preferencia de muchos profesores, en un sólo deporte.

El modelo horizontal quiere una visión distinta de la práctica y la enseñanza, su metodología parte de proporcionar un repertorio de habilidades "de base" y común a varios deportes, ejemplos de éstas son el concepto de deporte educativo, acuñado por Le Boulch (1991), y los juegos modificados (Devís, 1996).

La postura que presento se adhiere al modelo horizontal, en una derivación denominada modelo comprensivo, que considera a los juegos modificados como práctica sobresaliente.

Este modelo, en cuanto a su función, se desarrolla por la comprensión y para la comprensión, e implica una actividad de pensamiento enfocada a que los niños descubran la lógica de los juegos. Los juegos modificados se ubican en la frontera entre el juego libre y el deporte. En este tipo de juegos se exagera la táctica en vez de la técnica, y sus reglas se modifican de acuerdo con las edades de los niños. Trabajar con mayor énfasis cuestiones de la táctica de juego da paso al pensamiento estratégico, pues se comprende más la lógica del mismo.

El planteamiento de los juegos modificados tiene validez pedagógica en tanto que:

a. Promueven la participación de todos los niños, tanto los de poca o mucha habilidad, porque se reducen las exigencias técnicas del juego.

b. Integran la participación de niños y niñas en las mismas actividades, ya que si la técnica se deja de lado, se favorece como objetivo la formación de grupos mixtos y la participación activa de los muchachos y las muchachas.

c. Reducen la competitividad, ya que la intervención del profesor resalta la naturaleza comprensiva y dinámica del juego mismo.

d. Los niños participan en forma decisiva en el proceso de aprender, pues tienen la oportunidad de poner, quitar y cambiar las reglas de los juegos, con ello la posibilidad de estimular la creatividad y la invención para hacer incluso nuevos juegos modificados ${ }^{2}$ (Devís, 1996. pp. 49-50)

Sin entrar en más detalles, apunto las orientaciones peda-gógicas más significativas que, desde mi perspectiva, pueden animar la práctica de este tipo de juegos motores:

a) son actividades que no necesariamente son "antecedentes de los deportes"; 
b) tienen que ver con el control más específico de distintos patrones de movimiento en actividades que implican oposición e incertidumbre en un ambiente de juego, $y$

c) están orientados a la conquista y el manejo de trayectorias, desplazamientos, giros y manipulaciones, además de involucrar el pensamiento estratégico.

Con respecto a su fundamento didáctico, se apoyan en cuatro cuestiones básicas: el conocimiento sobre las acciones, la elaboración de esquemas de movimiento, la variabilidad de la práctica y en que los aprendizajes adquiridos suponen una transferencia, es decir, que la actividad corporal de tipo inteligente les permite a los niños establecer principios y relaciones entre unas habilidades y otras (Ruiz, 1994, p. 127).

\section{Los estilos de enseñanza}

Los estilos de enseñanza tienen que ver con el contexto de la práctica y con la valoración del contenido. Implican de forma protagónica la relación que establece el profesor entre el niño y su conducta, para que éste obtenga, o no, parte del conocimiento de saber cómo hacer los desempeños motores.

Mi postura acepta más la comprensión del proceso de aprender que la perniciosa "guerra de conceptos". De ahí que, en lo que llamo estilos de enseñanza, coincida con Delgado Noguera (1993), en tanto que los distintos estilos encierran de algún modo muchas de las relaciones didácticas y elementos personales. Así, quedan incluidos tanto los aspectos técnicos y comunicativos como los referentes a la organización del grupo, las relaciones afectivas que el docente adopta al momento de dirigir a los niños y el manejo del contenido. La serie de decisiones que el profesor toma con respecto a la dosificación de programas, la aplicación de un método en particular, la organización del grupo y la forma de relacionarse con los niños tienen que ver, pues, con un estilo de enseñanza. También incluyo en este espectro el patrón de conducta que sigue el profesor durante el ejercicio de la enseñanza.

De las distintas clasificaciones de estilos de enseñanza que hace el mencionado autor, me inclino y sostengo que para edificar el conocimiento del saber cómo en los niños se ha de centrar la atención en los estilos de enseñanza que comportan la implicación cognoscitiva directa del alumno en su aprendizaje. En estos estilos se encuentran el descubrimiento guiado; la resolución de problemas, y el planteamiento de situaciones tácticas (que presumiblemente están presentes en los juegos modificados). Las resoluciones didácticas de estos estilos de enseñanza se distinguen porque varían las relaciones interpersonales entre maestro-alumno y entre los alumnos. Los elementos didácticos implican y motivan a los niños a un aprendizaje activo, significativo, que los conduce a la indagación y a la experimentación motriz (Delgado, 1993).

Es importante incluir y decir que la implicación cognitiva requiere de la autogestión ${ }^{3}$ como un potente medio de relación entre el niño y su conducta y en el manejo del contenido, donde en forma personal el alumno regula y controla su actuación y resuelve los distintos problemas a partir del reconocimiento de sus propias capacidades, posibilidades y límites. Cabe mencionar que el niño no aprende sólo del manejo de los contenidos, sino por lo que hace con ellos; un ejercicio es más valioso por que permite construir, que el ejercicio en sí.

La autogestión en el niño es motivada por el profesor y su estilo de enseñanza porque incentiva la exploración y la solución de problemas motrices, por el descubrimiento guiado y un trabajo encaminado a atender la diversidad, donde poco a poco la percepción de sí (en los niños) vaya descubriendo posibilidades y alternativas de acción. 


\section{Bibliografía}

ARNOLD, P. J. (1991). Educación física, movimiento y currículum. Madrid. Morata.

BRITO, L. F. (1996). Educación Física y recreación. México: Editorial Edamex.

CONTRERAS, O. (1998). Didáctica de la educación física; un enfoque constructivista. Barcelona. Editorial INDE.

DELGADO, M. (1993). Los métodos didácticos en la educación física. En: Fundamentos de educación física para enseñanza primaria. Vol. II. (Compendio). Barcelona. Editorial INDE.

DEVIS, J. (1996). Educación física, deporte y currículum. Madrid. Visor.

DEVIS, J. y Pieró, C. (1992). Nuevas perspectivas curriculares en educación física: la salud y los juegos modificados. Barcelona. Editorial INDE.

\section{Notas}

1 El término flexibilidad lo utilizó en este caso con base en las consideraciones de Le Boulch (1991) que establece que el aprendizaje de los automatismos motores es de dos tipos: los rígidos y los plásticos. Los primeros hacen referencia a un automatismo que requiere una forma específica de ejecución, por ejemplo, las brazadas en natación, y los plásticos hacen referencia a automatismos que son capaces de responder a situaciones diversas, no especializados. Por tanto utilizo el término flexibilidad como similar al aprendizaje de automatismos plásticos.
DIAZ, J. (1994). El currículum de la educación física en la reforma. Barcelona. Editorial INDE.

GONZÁLEZ, M. (1993). La educación física: fundamentación teórica y pedagógica. En Fundamentos de educación física para enseñanza primaria. Vol. I. (Compendio). Barcelona. Editorial INDE.

LE BOULCH, J. (1991). El deporte educativo. España: Paidós.

RUIZ, L. (1995). Competencia motriz; elementos para comprender el aprendizaje motor en educación física escolar. Madrid. Editorial Gymnos.

RUIZ, L. M. (1994). Deporte y aprendizaje. Procesos de adquisición y desarrollo de habilidades. Madrid. Visor.

WALLON, H. (1964). Del acto al pensamiento: ensayo de psicología comparada. Buenos Aires. Lautaro.

2 También puede consultar Devís y Pieró (1992).

3 La autogestión la entiendo como el proceso personal de regulación y motivación de la conducta, donde por medio de la finalidad de la misma, un sujeto en lo particular resuelve las tensiones y desequilibrios tanto internos como externos, provocados por la motivación. Los elementos de la conducta son: motivación, finalidad, sentido y estructura. Cfr. Brito (1996, pp. 18-28). 